\title{
Proliferative diabetic retinopathy: predictive and preventive measures at hypoxia induced retinal changes
}

\author{
Tatjana Josifova $\cdot$ Ivna Plestina-Borjan • \\ Paul Bernhard Henrich
}

Received: 7 January 2010 /Accepted: 28 January 2010/Published online: 1 April 2010

(C) European Association for Predictive, Preventive and Personalised Medicine 2010

\begin{abstract}
Retinal vasculature changes in diabetic patients are most common cause of blindness among eye diseases. Numerous studies have explored the role of the agiogenic factors in the progression of diabetic retinopathy (DR). The balance between angiogenic and antiangiogenic factors has a determining role in the DR progression. Current treatment modalities include laser photocoagulation, intravitreal drug application, and pars plana vitrectomy (ppv). These maneuvers are employed with occurrence of advanced retinal changes. New diagnostic approaches can provide better information for the initial retinal changes thereby requiring a new DR classification and treatment guidelines. The results that are expected from Diabetic Retinopathy Clinical Research Network (DRCR) are at the level where prediction and prevention can not be made. Innovative molecular-imaging technology, can pave the way for application of novel clinical approaches. Identification of pathology-specific biomarkers and their application to diagnosis and treatment, support the individualized treatment algorithms.
\end{abstract}

The authors have full control of all primary data and they agree to allow the journal to review their data on request. None of the authors has a financial or proprietary interest in any material mentioned in the article.

T. Josifova $(\bowtie) \cdot$ P. B. Henrich

Department of Ophthalmology, University Hospital Basel,

Mittlere Strasse 91,

4031 Basel, Switzerland

e-mail: tjosifova@uhbs.ch

I. Plestina-Borjan

Department of Ophthalmology, University Hospital Split, Split, Croatia
Keywords Proliferative diabetic retinopathy · Innovative molecular-imaging technology $\cdot$ New classification and guidelines · Prediction/prevention at initial stages . Intravitreal drug application - Individualized treatment algorithms

\section{Prevalence and economic burden}

WHO has estimated that there were 171 million people worldwide with diabetes mellitus (DM) in 2000 and predicted that 366 million people will have DM by 2030. The United States Centers for Disease Control and Prevention have estimated that 13 million persons in the United States have diagnosed DM, and an additional 5,2 million have the disease but it is not yet being diagnosed [1].

Diabetes Mellitus is a severe metabolic disease associated with a number of complications including retinopathy, nephropathy and neuropathy. Mortality related to DM and its complications result in 3.8 million deaths annually, accounting for $6 \%$ of the total worldwide mortality $[2,3]$. The mortality risk in the diabetic population in the age of 40-49 years is about 6-7 years and for the age category of 60-69 it is about 3-4 years higher, compared to mortality risk, at the same age healthy population. The stage of nephropathy, including the severity of proteinuria is a predictable factor for the mortality risk. Approximately $2.5 \%$ to $15 \%$ of annual healthcare budgets is spent on care of patients with diabetes and associated complications. It has been estimated that programs to identify and treat diabetic retinopathy (DR) would have saved the United States health care budget nearly 400 million dollars annually in the early 1990s, ] a figure that would probably be substantially higher today [4]. 


\section{Consequences of "Bad Metabolic Memory"}

A lot of studies in DM type 2 between which is the UKPDS-Study, have underlined the beneficial effect of having the $\mathrm{HbAl} \mathrm{c}$, not $<6 \%$. A diverse array of micro- and macrovascular changes, are induced trough "Bad Metabolic Memory" and increase, as well as decrease of HbAlc (Fig. 1).

Retinal vascular changes in diabetic population are the most common cause of blindness among eye diseases. Impairment of retinal circulation results in blood flow alternations that affect the delivery of oxygen and metabolic substrates to the tissue. Various pathophysiological pathways mediate neuronal damage due to insufficient oxygen supply. Release of oxygen derived free radicals, nitric oxide-mediated neurotoxicity, and delayed cell death lead to neuronal damage. Poor glycemic control sets the stage for initiation of the microvascular changes [4].

The molecular basis of DR and maculopathy takes into consideration the role of growth factors and cytokines in the development of diabetic vascular alterations, their specific influence on the cellular interaction between retinal endothelial cells and pericytes, and the role of intravascular blood components. The earliest histological features of DR include neuroretinal damage, capillary basement membrane thickening, loss of pericytes and loss of endothelial cells. The hallmark of proliferative diabetic retinopathy (PDR) is neovascularization (NV) which occurs at the latter stages of the disease and can result in blindness; NV is the consequence of abnormal fibrovascular proliferations with subsequent bleeding and retinal detachment [5]. Numerous

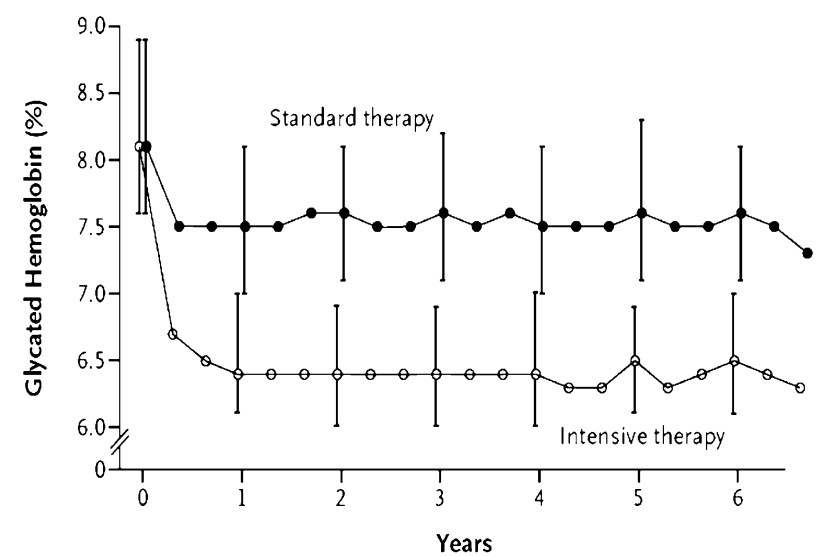

$\begin{array}{crrrrrrr}\begin{array}{l}\text { No. at Risk } \\ \begin{array}{c}\text { Standard } \\ \text { therapy }\end{array}\end{array} & 5109 & 4774 & 4588 & 3186 & 1744 & 455 & 436 \\ \begin{array}{c}\text { Intensive } \\ \text { therapy }\end{array} & 5119 & 4768 & 4585 & 3165 & 1706 & 476 & 471\end{array}$

Fig. 1 ACCORD - Study: HbA1c influence on cardiovascular complications. Increase of glycated hemoglobin in diabetic population at baseline, and in 6 years follow-up period, has the same impact on the cardiovascular complications, as the decrease of $\mathrm{HbA} 1 \mathrm{c}<6.0 \%$ studies have underlined the role of the angiogenic factors in the progression of DR and include vascular endothelial growth factor (VEGF), insuline-like growth factor (IGF-1), hepatocyte growth factor (HGF), basic fibroblast growth factor (b-FGF), platelet-derived growth factor (PDGF), pro/ inflammatory cytokines and angiopoetins. On the other hand, the major antiangiogenic factors are: pigment epithelium derived factor (PEDF), transforming growth factor beta (TGF-beta), thrombospondin (TSP) and somatostatin [5-7]. The balance among angiogenic and antiangiogenic factors has a determining role in the progression of DR.

\section{Benefits of early therapeutic measures}

The Early Diabetic Retinopathy Study (EDRS) demonstrates the ability of panretinal photocoagulation to reduce the rate of severe visual loss by $50 \%$ for eyes with high risk characteristics, defined as NV originating from optic disc $(>1 / 3$ disc diameter), any $\mathrm{NV}$ originating from the optic disc with hemorrhage, and NV originating from the retina with vitreous hemorrhage. The EDRS showed that patients with (type 2) DM who were older than 40 years of age, with severe nonproliferative DR (defined as hemorrhages in four quadrants, or intraretinal microvascular abnormalities in one quadrant), have benefited from early panretinal photocoagulation. The Early Vitrectomy Diabetic Study (EVDS) showed that early vitrectomy (within 6 months of onset of vitreous haemorrhage) was associated with better results in type 1 diabetes mellitus patients [7-9].

\section{Where are we now with our knowledge?}

Where are we now in relation to our knowledge in prevention and early treatment of DR? Did we follow our investigations in a direction that can give us solution of the main problems, or our investigations do not have a real clinical impact? Can we expect better predictive strategies for early diagnosis and treatment?

To answer these questions we will summarize our results achieved with currently available diagnostic and therapeutic approaches. These are exciting times for specialists in the management of retinal diseases. A number of advances in science have been translated into real and measurable advances in patient care.

The emerging investigations are concentrated on different secretory proteins and their role in the development on PDR. For example secreted acidic proteins rich in cystein (SPARC) are isolated from the vitreus fluid and are increased in patients with PDR. Furthermore, subretinal injections of recombinant SPARC adenovirus, induce PDR- 
like changes in the rat, thereby providing a relevant animal model for the investigation of PDR. [10, 11].

Robo4, a member of roundabout (Rabo) family, acts as a neuronal guidance receptor and plays some role in vasculogenesis and angiogenesis. Several studies have shown its effect on the formation of fibrovascular membranes in patients with PDR, and its role in choroids-retina endothelial $(\mathrm{RF} / 6 \mathrm{~A})$ and human retinal pigment epithelial cells. Silencing the expression of Robo4 in RF 6A and RPE cells inhibited their proliferation and reduced the hypoxic condition tolerance [12].

A number of studies have estimated increased levels of interleukin 8 in vitreous samples from patients with PDR and in patients with more severe large-vessel gliotic obliteration [13].

Cystein-rich 61 (Cyr61) induces are reported to mediate angiogenesis, and they have been shown to cause proliferation, migration, and synthetic matrix tube formation of $\mathrm{RF} / 6 \mathrm{~A}$ cells. Hypoxia significantly induced Cyr61 mRNA and protein expression. Recent reports indicate that Cyr61 acts as an angogenic mediator of ocular $\mathrm{NV}$ in vitro and in vivo. It may interact in synergy with VEGF in the pathogenesis of PDR [14]. On the other hand, rosiglitazone maleate, an oral peroxsisome-proliferating activated receptor gamma agonist and oral insulin sensitising agent with potential antiangiogenic activity, delays onset of PDR [15].

The "Golden Standard" of therapy in DM is insulin, and previous studies have emphasized its inhibitory effect on the progression of the DR. However, recent information suggests this to be controversial because insulin can stimulate VEGF and hypoxia-inducible factor-1 (HIF-1) expression in retinal pigment epithelial cells. VEGF is considered as a main factor for the development of the PDR, but recently, a $634 \mathrm{C} / \mathrm{G}$ polymorphisms in the VEGF gene was shown to be associated with PDR. The expression of VEGF, VEGFR1 and VEGFR2 levels is much higher in patients with PDR and DM, than in diabetics with PDR and DM type 2 [16].

Studies that have analyzed the expression of integrins and their localization within the endothelium (in membranes from proliferative DR isolated during the vitreoretinal surgery) may provide new possibilities for the future treatment of the proliferative DR. The results suggest an essential role of integrins alfa and beta 3 in the pathogenesis of PDR.

A number of studies have shown the beneficial effect of intravitreal Anti-VEGF therapy [17]. The results were achieved between 1-3 weeks in the patients with retinal neovascularization elsewhere and neovascularization of the disc. Assessment of the short-term safety and efficacy of inravitreally administrated bevacizumab (an Anti-VEGF agent) as an adjunctive treatment for PDR, especially in the severe cases with iris $\mathrm{NV}$, has been estimated in a number of studies [17-20]. These studies confirmed regression of the NV, but the main disadvantage of the Anti/VEGF drug administration is the need of repeated injections in order to prevent recurrences of the NV [21].

Glucocortcoids (e.g., triamcinolon acetonide) that are among the drugs used for intravitreal application can influence diabetic macular edema (DME). The pathogenesis of PDR is multifactorial involving both angiogenic and inflammatory processes. When administrated together with Anti-VEGF drugs (bevacizumab, ranibizumab) can induce $\mathrm{NV}$ and DME regression. The results achieved from the studies may make this procedure an important adjunctive treatment in the management of selected cases with severe PDR [22].

The long-term results of intravitreal bevacizumab (Avastin), alone, in patients with PDR do not reveal any safety concerns $[22,23]$.

Current treatments for conditions such as DME are limited. For instance, laser photocoagulation, while superior to intravitreal triamcinolone (IVT) or Anti-VEGF drugs, reduces but does not eliminate the risk of continued visual loss and is destructive to the retina. Focal/Grid laser is more effective than IVT with fewer side effects within the initial 2 years. Laser or IVT are both more likely to improve the VA over three years compared to the expected untreated course. Focal/Grid laser should serve as benchmark for future clinical trials for DME.

Clinical investigations include those which evaluated the efficacy of intravitreal injection of bevacizumab in preventing panretinal photocoagulation, macular thickening and visual dysfunction in eyes with severe PDR. The results showed that the best corrected visual acuity increased after the combined AntiVEGF-laser treatment while the central foveal thickness decreased. [22, 23].

Intravitreal injection of bevacizumab can also be used in patients with PDR, that are undergoing pars plana plana vitrectomy (ppv). The intravitreal administration of bevacizumab (before ppv for PDR) facilitates surgery and may decrease the rate of postoperative vitreous hemorrhage and improve visual acuity [24, 25]. Ranibizumab (Lucentis) is an affinity-matured recombinant humanized immunoglobulin monoclonal antibody fragment with a molecular weight of $48 \mathrm{kD}$ that binds to and inhibits the biologic activity of all isoforms of human VEGF by preventing interaction with its receptors. The fragment is one third the size of a fulllength antibody and readily penetrates all layers of the retina after intravitreal injection, consequently decreasing cell proliferation and vascular permeability. The latest studies have also shown the positive effect of the drug, applied intravitreal in cases of DME.

The DRCR Network makes a collaborative effort on DR and additional retinal disorders. The funding was through National Eye Institute that sponsored cooperative agree- 
ment that was initiated in September 2002. At the moment there are eleven "on-going" protocols that should estimate the results of different therapeutic approaches in cases of DR. The objective is to develop a collaborative network to facilitate multicenter clinical research on DR, DME and associated conditions (Fig. 2).

The goals of the DRCR are:

$>$ Involvement of community based practices as well as academic or university-based centres.

$>$ Collaborate with industry to facilitate investigations and pursue opportunities otherwise not possible and to do so in a manner consistent with the Networks dedication to academic integrity and optimal clinical performance.

Currently 8 trails are being planned. This will include the effects of cataract surgery upon DME and the effects of intravitreal plasmin to cause a posterior vitreous detachment upon DME.

With the advent of new sophisticated techniques and diagnostic tools, the latest classification of the DR seems to be insufficient. Initial diabetic changes can not be detected with the current diagnostic methods. Thus a new classification for diabetic retinal changes should be considered. Triggered on the molecular level, the non-responders to the standard therapy should be provided a strategy of personalized patient treatment. Based on emerging pathophysiolgical mechanisms, we should devise novel therapies and planning. Although individualized therapy planning seems to be expensive, we should consider the fact that the diabetic complications lead to economic and psycho-social problems. Diabetes costs range from $2.5 \%$ to $15 \%$ of annual health care budgets. The complicated treatment phase, including the sophisticated and expensive techniques of ppv, using different kind of drug substitutes do not always provide us satisfactory results. The main problem at this point is not only the associated complications of the treatment, but the chronicity of the disease. The long-term results of the surgery are not always stabile over time, and a lot of late complications can not be avoided. All these facts, lead us to a "Point of no Return". Thus there is a need for new diagnostic and therapeutic approaches to be instituted much earlier at a time when there is no evidence of retinal damage. Hypertension, insulin resistance, dyslipidemia and obesity, are commonly found in a combination with this stage of PDR. New diagnostic images, that can discover early diabetic changes, can provide useful informations for the novel DR treatment. Pathology specific biomarkers, DNA analysis in conditions of chronic diseases diagnostic and treatment, do not deliver us only information about the general concept of treatment, but also help us in the individual-treatment, individual drug response and benefits according to this strategy of individual healthcare. There is a need of collaborative network, to facilitate multicenter clinical activities, and thereby development of advanced treatment algorithms for DR and DME.

The multidisciplinary approach to chronic diseases, such as DR can initiate research activities in different fields that could create the model of personal-based-patient-care. This means, we should gain new strategies to the optimal concept that includes:

- Recognize the population at risk of the disease

- Follow-up before the initial changes become manifested

- Search for new approaches that can prevent the development of the disease

- Early treatment

- Optimal follow-up
Fig. 2 Searching for the results of different treatment modalities in cases of diabetic macular edema, according to the DRCR Network protocols

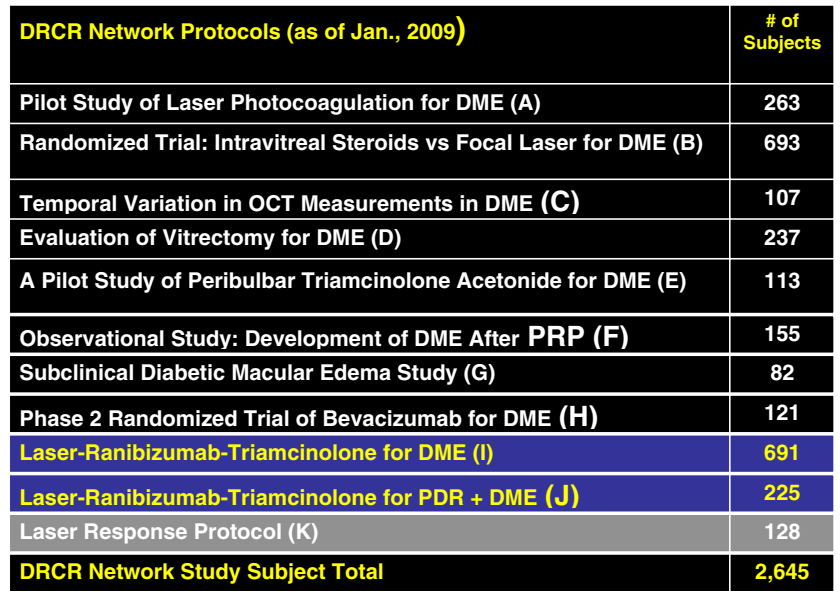




\section{References}

1. American Academy of Ophthalmology. 2002. http://www.aao.org

2. Adamiec-Mroczek J, Oficjalska-Mlynczak J, Misiuk-Hoilo M. Proliferative diabetic retinopathy-The influence of diabetes control on the activation of the intraocular molecule system. Diabetes Res Clin Pract. 2009;84(1):46-50.

3. Lewis H, Ryan RS. Medical and Surgical Retina. London: Mosby; 1994.

4. Gunduz K, Bakri SJ. Management of proliferative diabetic retinopathy. Compr Ophthal Update. 2007;8(5):245-56.

5. Simo R, Carrasco E, Garcia-Ramirez M, et al. Angiogenic and antiangiogenic factors in proliferitive diabetic retinopathy. Curr Diabetes Rev. 2006;2(1):71-98.

6. Ning A, Cui J, Maberley D, et al. Expression of integrins in human proliferative diabetic retinopathy membranes. Can $\mathrm{J}$ Ophthalmol. 2008;43(6):683-8.

7. Praidou A, Klangas I, Papakonstantinou E, et al. Vitreous and serum levels of platelet-derived growth factor and their correlation in patients with proliferative diabetic retinopathy. Curr Eye Res. 2009;34(2):152-61.

8. Fujioka S, Karashima K, Saito Y. Differences of ocular circulation in eyes with proliferative diabetic retinopathy after panretinal photocoagulation with and without rubeosis iridis. Nippon Ganka Gakkai Zasshi-Acta Societatis Ophthalmologicea Japonicae. 2009;113(1):11-5.

9. Vernon SA, Bhagey J, Boraik M, et al. Long-term review of driving potential following bilateral panretinal photocoagulation for proliferative diabetic retinopathy. Diabet Med. 2009;26(1): 97-9.

10. Nakamura S, Iwasaki N, Funatsu H, et al. Impact of variants in the VEGF gene on progression of proliferative diabetic retinopathy. Graefes Arch Clin Exp Ophthalmol. 2009;247(1):21-6.

11. Watanabe K, Okamoto F, Yokoo T, et al. SPARC is a major secretory gene expressed and involved in the development of proliferative diabetic retinopathy. J Atheroscler Thromb. 2009;16 (2):69-76

12. Huang L, Yu W, Li X, et al. Expression of Robo4 in the fibrovascular membranes from patients with proliferative diabetic retinopathy and its role in RF/6A and RPE cells. Mol Vis. 2009;15:1057-69.
13. Petrovic MG, Korosec P, Kosnik M, et al. Vitreous levels of interleukin- 8 in patients with proliferative diabetic retinopathy. Am J Ophthalmol. 2007;143(1):175-6.

14. You JJ, Yang CH, Chen MS, et al. Cysteine-rich 61, a member of the CCN family, as a factor involved in the pathogenesis of proliferative diabetic retinopathy. Investig Ophthalmol Vis Sci. 2009;50(7):3447-55.

15. Shen LQ, Child A, Weber GM, et al. Rosiglitazone and delayed onset of proliferative diabetic retinopathy. Arch Ophthalmol. 2008;126(6):793-9.

16. Zhang P, Liu N, Wang Y. Insulin may cause deterioration of proliferative diabetic retinopathy. Med Hypotheses. 2009;72(3):306-8.

17. Mason 3rd JO, Yunker JJ, Vail R, et al. Intravitreal bevacizumab (Avastin) prevention of panretinal photocoagulation-induced complications in patients with severe proliferative diabetic retinopathy. Retina. 2008;28(9):1319-24.

18. Moradian S, Ahmadieh H, Malihi M, et al. Intravitreal bevacizumab in active progressive proliferative diabetic retinopathy. Graefes Arch Clin Exp Ophthalmol. 2008;246(12):1699-705.

19. Arevalo JF, Wu L, Sanchez JG, et al. Intravitreal bevacizumab in active progressive proliferative diabetic retinopathy: 6-months follow-up. Eye. 2009;23(1):117-23. 22.

20. Mason 3rd JO, Nixon PA, White MF. Intravitreal of bevacizumab (Avastin) as adjunctive treatment of proliferative diabetic retinopathy. Am J Ophthalmol. 2006;142(4):685-8. 23.

21. Oshima Y, Sakaguchi H, Gomi F, et al. Regression of iris neovascularization after intravitreal injection of bevacizumab in patients with proliferative diabetic retinopathy. Am J Ophthalmol. 2006;142(1):155-8. 25.

22. Arimura N, Otsuka H, Yamakiri K, et al. Vitreos mediator after intravitreal of bevacizumab or triamcinolone acetonide in eyes with proliferative diabetic retinopathy. Ophthalmology. 2009;116 (5):921-6. 25.

23. Thew M. Rapid resolution of severe retinal neovascularization in proliferative diabetic retinopathy. Clin Exp Optom. 2009;92 (1):34-7.

24. Ishikava K, Hinda S, Tsukahara $\mathrm{Y}$, et al. Preferable use of intravitreal bevacizumab as a pretreatment of vitrectomy for severe proliferative diabetic retinopathy. Eye. 2009;23(1):108-11.

25. Park SP, Ahn JK, Lee GH. Morphologic changes in the anterior segment after phacovitrectomy for proliferative diabetic retinopathy. J Cataract Refract Surg. 2009;35(5):868-73. 